\title{
Botulinum A toxin as a treatment of detrusor- sphincter dyssynergia in patients with spinal cord injury: MRI controlled transperineal injections
}

Brigitte Schurch, Juerg Hodler, Bilijana Rodic

\begin{abstract}
Objectives-To correlate clinical and urodynamic findings with MRI in patients with spinal cord injury and detrusorsphincter dyssynergia who were consecutively treated with transperineal injections of botulinum-A toxin (BTX-A) under EMG control.

Methods-Six patients with spinal cord injury and upper motor neuron bladder dysfunction associated with detrusorsphincter dyssynergia were prospectively analysed. One hundred international units (IU) BTX-A (Botox ${ }^{\circledR}$ in $1 \mathrm{ml}$ normal saline without preservative) diluted 1 to 1 with $1 \mathrm{ml}$ gadopentetate were injected transperineally under EMG control. MRI was started immediately after needle withdrawal.

Results-In all six patients gadopentetate was located in the external urethral sphincter on MRI. In no patient did traces of gadopentetate appear in the perineal musculature located in the vicinity of the external urethral sphincter. No patient developed resistance to BTX-A. All patients showed an (ongoing) improvement of their voiding function after BTX-A injections.

Conclusions-Transperineal injections of BTX-A under EMG control are efficient in the release or amelioration of lower urinary tract obstruction due to detrusor sphincter dyssynergia in patients with spinal cord injury. Despite well described methods, EMG of the external urethral sphincter is difficult and it is not possible to definitively exclude false recordings of the surrounding perineal musculature. $B y$ the use of MRI it was shown that both the EMG recordings and transperineal injection method are precise.
\end{abstract}

(F Neurol Neurosurg Psychiatry 1997;63:474-476)

Keywords: Botulinum toxin; neurogenic voiding disorders; detrusor-sphincter dyssynergia; electromyography; magnetic resonance imaging

Detrusor-sphincter dyssynergia represents a major cause of voiding dysfunction in upper motor neuron bladder. With failure of conservative management of the bladder, sphincterotomy remained until recently the only alternative treatment. Despite improvements in the technique, complications are common.
High reoperation rates and significant incidence of long term clinical failures have been described. ${ }^{12}$ Botulinum A (BTX-A) injections into the external urethral sphincter represent a valuable alternative to surgical sphincterotomy. ${ }^{3}$ The effects of BTX-A toxin on neurogenic detrusor-sphincter dyssynergia were prospectively analysed between 1988 and 1994 in 24 patients with complete spinal cord injuries. Fifty transurethral and 35 transperineal BTX-A injections were applied during 38 individual treatments in the 24 patients. Transurethral injections were made through a cystoscope and transperineal injections under EMG control as described by Dyro. ${ }^{4}$ Twenty one out of 24 patients showed a significant improvement of their voiding function after treatment. Mean maximal urethral pressure during detrusor-sphincter dyssynergia, duration of detrusor-sphincter dyssynergia, and basic urethral pressure during detrusor-sphincter dyssynergia decreased by $48 \%, 47 \%$, and $20 \%$ respectively (paired student's $t$ test, $\mathrm{P}<0.01$ ). Detrusor-sphincter dyssynergia disappeared in eight patients and improved in 13 patients. Increased incontinence after treatment was never seen. Some of the patients were already wearing a condom, the main goal of the treatment being to decrease the voiding pressure. In continent patients, improved voiding led to a larger free interval between micturitions. A protocol of 1 injection of 100 international units (Botox ${ }^{\circledR}$, Allergan Inc, Irvine California) diluted in $1 \mathrm{ml}$ normal saline without preservative repeated three times at monthly intervals improved symptoms for at least nine months. Both transurethral and transperineal injections were efficient in suppressing or ameliorating detrusor-sphincter dyssynergia. Transperineal injections are easier to perform for neurologists taking care of patients with spinal cord injury who are not trained to perform cystoscopies. Despite well described EMG methods it cannot be ruled out that BTX-A is injected elsewhere in the perineum. To confirm that our injection method was correct, a prospective study was performed in six patients with spinal cord injury treated with transperineal injections of BTX-A.

Patients and methods

Three paraplegic (two complete, one incomplete) and three tetraplegic (two complete, one incomplete) patients were included in the study. Out of these six patients, five were 


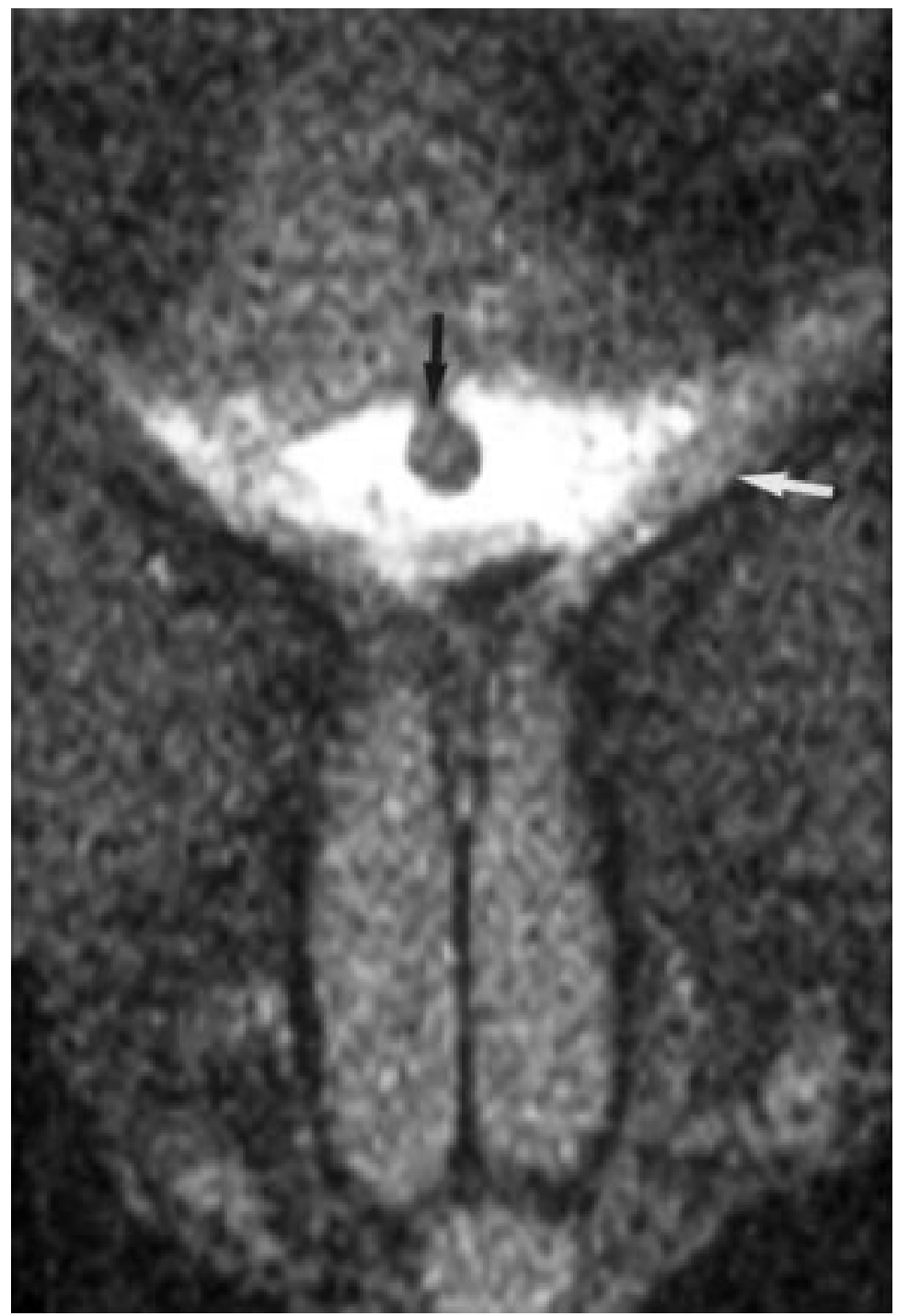

Complete paraplegic patient T4 with upper motor neuron bladder and detrusor-sphincter dyssynergia. MRI after transperineal injection of BTX-A with gadopentetate. Fat suppressed T1 weighted MRI axial slice at the level of the inferior fascia of the urogenital membrane. Gadopentetate (high signal) is seen uniformely distributed in the external urethral sphincter all around the urethra (black arrow). No trace of gadopentetate appears in the perineal musculature located in the vicinity of the external urethral sphincter (white arrow shows $m$ transversus profondus).

known to have been successfully treated with BTX-A injections during the first study. In these patients injections were repeated nine to 12 months after the last injection of the first study to maintain efficacy of the treatment. One patient was included new to the treatment protocol. A $26 \times 2$ gauge polytetrafluoroethylene (Teflon) coated monopolar needle electrode (Dantec, Bollinger AG, Zurich, Switzerland) was inserted in the midline 1.5 to $2.0 \mathrm{~cm}$ anterior to the anus. A gloved finger in the rectum monitored the position of the prostate while the electrode was directed toward the apex. Final localisation was confirmed by monitoring the needle motor unit activity electromyographically (Amplaid, Italy) and in three patients by consecutive examination of the needle position under an image intensifier. One hundred international units (IU) of BTX-A toxin $\left(\right.$ Botox ${ }^{\circledR}$, in $1 \mathrm{ml}$ normal saline without preservative) diluted 1 to 1 with $1 \mathrm{ml}$ gadopentetate (Magnevist ${ }^{\circledR}$, Schering, Berlin) were injected transperineally. In three patients the gadopentetate concentration was $0.5 \mathrm{~mol} / 1$. This concentration led to artifacts, which did not interfere with the diagnosis. In the next three patients gadopentetate concentrations of $4 \mathrm{mmol} / \mathrm{l}, 50 \mathrm{mmol} / \mathrm{l}$, and $100 \mathrm{mmol} / 1$ were employed. The injections were performed in the MR suite, outside the magnet and MRI was started immediately after needle withdrawal. Images were obtained on a $1.0 \mathrm{~T}$ Siemens Impact scanner (Siemens Medical Systems, Erlangen, Germany), with a receive only coil dedicated to imaging of the spine. Sagittal and axial images covering the urethra from the bladder neck to the spongious urethra were obtained. Fat suppressed T1 weighted images with the following indices were found to be optimal for our purpose: repetition time 541 $\mathrm{ms}$, echo time $15 \mathrm{~ms}$, field of view $240 \mathrm{~mm}$, image matrix $200 \times 256$, slice thickness, interslice gap $0.5-2.5 \mathrm{~mm}$. One acquisition was employed, and 13 slices were obtained.

\section{Results}

In all six patients gadopentetate was located in the area of the external urethral sphincter on MRI. On axial slices gadopentetate could be seen all around the urethra in four patients (figure) or predominating on one side in two patients. A hypointense artifact was found within the zone of hyperintensity after the injection of undiluted gadopentetate. A total of $50 \mathrm{mmol} / 1$ or $100 \mathrm{mmol} / 1$ concentrations of gadopentetate (before further dilution by Botox $\left({ }^{\circledR}\right)$ seemed to provide optimal contrast for our purpose, without producing artifacts. In no case did traces of gadopentetate appear in the perineal musculature located in the vicinity of the external urethral sphincter. In the five pretreated patients, one single reinjection of BTX-A allowed the efficacy of the treatment to be maintained. In the last case, BTX-A treatment was just started, two more injections being needed before re-evaluation of the voiding function.

\section{Discussion}

Twenty one of our 24 patients with spinal cord injury treated previously by transurethral or transperineal injections of BTX-A showed statistical improvement of their sphincter dysfunction. ${ }^{3}$ Three injections at one month intervals provided long term (nine to 12 months) efficiency. With transperineal injections, EMG controls are necessary to secure localisation of the external urethral sphincter. However, it is debatable whether EMG recordings really disclose the activity of the external urethral sphincter and not of the surrounding perineal musculature. In this case efficacy of the treatment would be doubtful. Using MRI we could show that our injection technique is correct as gadopentetate was seen located around the urethra in the external urethral sphincter and not in the perineal musculature. Despite asymmetric localisation of the contrast medium in some patients, urodynamic controls showed an ongoing improvement of external urethral sphincter function. It can be assumed 
that BTX-A diffused all around the external urethral sphincter or that partial weakening of the external urethral sphincter was sufficient to ameliorate detrusor-sphincter dyssynergia. As side effects never occurred, it is likely that the high affinity of BTX-A for skeletal muscle end plates minimises the risks of systemic contamination. On the other hand, high resorbtion of gadopentetate from the surrounding venous plexus could be seen on MRI in all our patients. None of our patients developed resistance to BTX-A. Therefore, it can be assumed that they did not become immune to BTX-A. Consequently, no hypersensitivity tests were done.

\section{Conclusion}

Botulinum A toxin has been shown to constitute a valuable alternative management of bladder dysfunction with detrusor-sphincter dyssynergia in patients with spinal cord injury who cannot perform self catheterisation or do not desire surgery. ${ }^{3}$ Both transperineal and transurethral injections are equivalent in giving efficient release or amelioration of the lower urinary tract obstruction secondary to detrusor-sphincter dyssynergia. Transperineal injections are easier to perform by neurologists who are not used to performing cystoscopies but are trained in EMG. Despite well described methods, ${ }^{4}$ EMG of the external urethral sphincter is difficult and it is not possible to definitively exclude false recordings of the sur- rounding perineal musculature. By the use of MRI we were able to show that both our EMG recordings and the transperineal injection technique are precise, opening the way for others to use this method of treatment of detrusor-sphincter dyssynergia. This explains why amelioration of the detrusor-sphincter dyssynergia symptoms occured in more than $85 \%$ of our patients. ${ }^{3}$ It is the first time that MRI controlled injections of BTX-A have been reported. Exact placement of the injection needle is important to achieve the desired therapeutic goals. Checking of the distribution of the BTX-A solution diluted with gadopentetate through MRI enables confirmation of accuracy or modification of the injection technique. This is of great importance in patients in whom BTX-A injections are used to relieve spasticity of deeply located muscles-for example, the external urethral sphincter.

This work was partially funded by the International Institute for Research in Paraplegia (P17/94-97).

1 Vapnek JM, Couillard DR, Stone AR. Is sphincterotomy the best management of the spinal cord injured bladder ? f Urol 1994;151:961-4.

2 Lockhardt JL, Vorstman B, Weinstein D, Politano VA. Sphincterotomy failure in neurogenic bladder disease. $\mathcal{f}$ Urol 1986;135:86-9.

3 Schurch B, Hauri D, Rodic B, Curt A, Meyer M, Rossier AB. Botulinum-A toxin as a treatment of detrusorsphincter dyssynergia: a prospective study in 24 patients with spinal cord injury. F Urol 1996;155:1023-9.

4 Dyro FM. Electrophysiology of the lower urinary tract. In: SV Yalla, EJ McGuire, A Elbadawi, J Blaivas, eds. Neurourology and urodynamics. Principles and practice. New York: Macmillan, 1989;1:45-58. 